\title{
General Multilayer Heat Transfer Model for Optical-Based Thermal Characterization Techniques
}

\author{
Xuhui Feng, Charlie King and Sreekant Narumanchi* \\ National Renewable Energy Laboratory, Golden, CO \\ * Email: sreekant.narumanchi@nrel.gov; Phone: 303-275-4062
}

\begin{abstract}
Optical-based techniques have been used to characterize thermal energy transport in materials for a few decades. To implement these techniques, a modulated heat source (either pulse or continuous wave) is always employed to excite a periodic temperature variation, which subsequently causes variation of temperature within the material and various other parameters that are a function of temperature. Ambient pressure, surface infrared properties, and radiation are all affected by the temperature variation and serve as indicators to indirectly detect the temperature change. To extract the properties of interest, theoretical models and solutions are necessary. In this work, we propose a general heat transfer model in multilayer structures. We also derived general solutions in the frequency domain using recursive matrix relationships. The recursive matrix simplifies the heat transfer analysis by only considering key parameters within the adjacent layers. In addition, the general analytical solution is only composed of a group of equivalent resistances that yield the contribution to the overall phase shift from each interface and can be used to directly calculate the phase difference within similar configurations. We applied this model and the associated solutions to analyze the data from the phase-sensitive transient thermoreflectance (PSTTR) technique. In contrast with typical thermoreflectance techniques, in the PSTTR technique, the pump and probe beams are applied on the opposite surfaces of the sample. We conducted PSTTR measurements on different multilayer structures, and then determined the thermal/physical properties of interest by fitting the theoretical solutions to the experimental data. The thermal conductivity of thermal grease (TC-5022) was determined to be $3.5 \mathrm{~W} /(\mathrm{m} \cdot \mathrm{K})$. Where appropriate, the fitted results were in excellent agreement with results from the literature, which validates this general model and the solution methodology. As another example of a multilayer structure, a novel direct-bonded interface that contains four layers, was studied.
\end{abstract}


Its overall thermal resistance was $0.46 \mathrm{~mm}^{2} \cdot \mathrm{K} / \mathrm{W}$, including the $\mathrm{Al}-\mathrm{Al}$ contact resistance of 0.33 $\mathrm{mm}^{2} \cdot \mathrm{K} / \mathrm{W}$ and $\mathrm{Al}-\mathrm{Si}$ contact resistance of $0.06 \mathrm{~mm}^{2} \cdot \mathrm{K} / \mathrm{W}$. Using this general model along with the PSTTR technique, an in-depth understanding of the interfacial resistance was achieved by investigating the contributions from each component in the interface.

Keywords: multilayer structure, heat transfer, thermoreflectance, phase shift, thermal resistance, direct bonding 


\section{Nomenclature}

Greek symbols

$\begin{array}{ll}\alpha & \text { Thermal diffusivity } \\ \omega & \text { Angular frequency } \\ \lambda & \text { Hankel transform variable } \\ \Phi & \text { Phase shift } \\ \rho & \text { Density }\end{array}$

Latin symbols

$\begin{array}{ll}A_{\mathrm{i}}, B_{\mathrm{i}} & \text { Coefficients of transformed solutions } \\ b & \text { Coordinates along the thickness direction } \\ \text { Sinh, Cosh } & \text { Hyperbolic functions in the transformed solutions } \\ c & \text { Specific heat } \\ d & \text { Thickness of each layer within the structures } \\ D & \text { Coefficient matrix } \\ f & \text { Frequency } \\ f(r) & \text { Axial symmetric heating function } \\ h & \text { Thermal conductance } \\ i & \text { index number of each layer } \\ j & \text { Imaginary unit } \\ k & \text { Bulk thermal conductivity } \\ L_{\mathrm{p}} & \text { Penetration depth } \\ p & \text { Parameter of interest in sensitivity analysis } \\ q_{0} & \text { Heat flux } \\ & \text { Radial coordinate }\end{array}$




$\begin{array}{ll}R & \text { Thermal resistance } \\ R_{L \mathrm{p}} & \text { Equivalent thermal resistance } \\ \mathcal{R} \mathcal{R} & \text { Lumped parameter to simplify the general solution of phase shift } \\ S_{\mathrm{p}} & \text { Sensitivity of variable } \\ S & \text { Laplace transform variable } \\ t & \text { Time } \\ T & \text { Temperature } \\ w & \text { Hankel transform of temperature } T \\ z & \text { Vertical coordinates along the thickness direction }\end{array}$

Subscripts

$1,2,3$

Index number of layers within the structure

$n$

Index number of arbitrary layer within the structure 


\section{INTRODUCTION}

With the rapid development of microelectronics/microelectromechanical systems, significant efforts have been put into exploring effective thermal management strategies to remove excessive heat that can degrade or damage components/devices. The heat removal path in electronics packaging usually involves multiple layers. These layers and the associated contact resistances can impose a significant resistance to the heat flow. A typical method for decreasing the contact thermal resistance and enhancing heat removal efficiency is the use of thermal interface materials/bonded interface materials (TIMs/BIMs) to fill in the voids/gaps between mating surfaces, thereby improving the contact and assisting the heat transfer [1-3]. It is important to understand and quantify the thermal performance of TIMs/BIMs to accurately determine the performance of electronic components and configurations. Therefore, a variety of techniques have been developed along with the heat transfer analysis to characterize TIMs/BIMs. For instance, the representative steady-state ASTM test stand [4] is widely used to determine both contact resistance and thermal conductivity based on the knowledge of temperature gradient and the heat fluxes in the test domain. In addition to the steady-state technique, transient techniques have become increasingly popular to characterize small scale materials that are extensively used in micro/nano-electronics, with advantages in the response time, precision, and spatial resolution. Typical transient techniques include flash [5] , 3-omega ( $\omega)$ [6], transient electrothermal (TET) [7-9], and a large group of optical-based techniques, including thermoreflectance (TTR) [10, 11], photothermal [12], and photoacoustic techniques [13, 14]. Compared with traditional transient techniques, optical-based transient thermal characterization techniques are non-contact/non-destructive and feature high precision in both the time and frequency domains.

To implement optical-based techniques to determine thermal/physical properties, theoretical models of heat conduction in solids are needed. Comparison between theoretical solutions and experimental data is key to the determination of properties. Heat conduction in solids under single or multiple heating sources has been extensively studied and reported. Carslaw and Jaeger [15] presented the solution of heat flow in 
a semi-infinite solid with the surface temperature imposed as harmonic function of time. Ohsone et al.'s [16] and Tong et al.'s [17] work further analyzed the application of this solution in the thermoreflectance technique with modulated heat sources. Fujii et al. [13] derived the photoacoustic theory for a two-layer model and extended the solution to multilayer structures. In multilayer structures, $\mathrm{Hu}$ et al. [14] also studied general photoacoustic effects in multilayer materials and used matrix methods to illustrate the recursive relationship between the coefficients of the adjacent layers. Solutions involving coefficient matrices are strongly preferred when solving heat transfer problems in multilayer structures with repeated interfacial boundary conditions. It consolidates similar thermal transport at various interfaces into recursive algebraic expressions, thereby reducing the tedious work of treating each layer individually. Iravani and Wickramasinghe [18] reported their work on the thermal wave propagation in isotropic and homogeneous materials using the scattering matrix approach and the expressions for the reflection and transmission coefficients at thermal discontinuities inside materials. Analogous to optical heating, Anderson [19] developed a method to calculate the laser heating on layered structures using Fourier transforms and matrix expressions. Madison and McDaniel [20] implemented a compact recursive $N$ layer Green's function to solve the coupled heat transfer problem in the thermal media. Then the function was used to study a particular case by fitting solutions to the experimental data for magneto-optical storage media. McGahan and Cole [12] also applied the Green's function to study the heat conduction in multiple layers for photothermal deflection experiments. A tri-diagonal matrix equation that governs the multilayer normal boundary fluxes was then derived. Feldman [21] developed a general iterative algorithm and also presented an approach for treating the interfaces under a one-dimensional modulated heating condition. The algorithm also involves a matrix to calculate the diffusion through boundaries and therefore incorporates the interface thermal resistance. This method was adopted in the time-domain and frequency-domain thermoreflectance technique in Cahill's work [22], which used Feldman's iterative algorithm to solve heat transfer problems in cylindrical coordinates with modulated surface heating. While most research on heat diffusion in multilayer structures assumes a single modulated heating source, 
Hao [23] presented analytical modeling to investigate the temperature distribution in arbitrary layers using multiple heaters.

Despite the various heat transfer models and analytical solutions, it is still difficult to practically apply them due to the complicated mathematical functions and inverse transforms involved. Proper assumptions or particular conditions are usually required to convert the solutions into applicable forms. Numerical integration is an alternative method that can be used to obtain the temperature field/distribution within the structure. But the numerical results lack insights into the underlying mechanisms and physics. In addition, the common assumption in these theoretical analyses that the substrate is semi-infinite or "thermally thick" is only valid for certain optical-based techniques and is not applicable to techniques that involve heat diffusion over a finite thickness, such as the phase-sensitive transient thermoreflectance (PSTTR) technique [17, 24], which applies two beams of lasers on opposite surfaces and is capable of characterizing microscale or sub-microscale TIMs/BIMs. In this work, we develop a general model for heat transfer within multilayer structures with finite thicknesses and a single heat source. With specific conditions and assumptions, we solve the proposed heat diffusion equation using recursive matrices and mathematical transforms and also complete the inverse transform to simplify the solutions into a concise analytical form without complicated functions. This analytical solution not only indicates that the phase shift is a cumulative effect of the thermal wave propagation, but also quantitatively and explicitly presents contributions from each layer and interface to the overall thermal resistance. In Section 2, the onedimensional (1-D) general models describing the heat transfer in the multilayer structures with different number of layers is introduced with necessary mathematical derivations. Then, the principle of the PSTTR technique and the experimental apparatus are presented in Section 3, with a brief discussion of the samples that are measured and analyzed. The last section includes a discussion of the key experimental results. Uncertainty and sensitivity analyses are also included in Section 4 to show the influence of different parameters on the experimental results. 


\section{GENERAL HEAT TRANSFER MODEL IN A MULTILAYER STRUCTURE}

For optical-based techniques, modulated optical signals are commonly used as a heat source to excite temperature variation in the sample under test. For example, ultrafast laser pulses (with frequency in the megahertz range) are applied to study the non-equilibrium heat transfer processes on the order of picoseconds. Under a modulated heat source, the thermal penetration depth $\left(L_{\mathrm{p}}\right)$ is estimated as $\sqrt{2 \alpha / \omega}$, where $\alpha$ is the thermal diffusivity and $\omega$ is the angular frequency of the modulation. The penetration depth denotes a length scale over which the thermal energy can propagate before getting damped by $63 \%$. A material is defined as "thermally thick" [25] when its thickness is significantly greater than the thermal penetration depth. For most materials subjected to measurements within the $100-10^{6} \mathrm{~Hz}$ frequency range, the thermal penetration depth is anywhere from a few hundred nanometers to a few hundred micrometers. Penetration depth also determines the limit of the spot size $\left(r_{\text {spot }}\right)$ of the optical signal in optical-based measurements. The ratio between $L_{\mathrm{p}}$ and $r_{\text {spot }}$ determines whether the heat source is a planar or point source, and whether the heat transfer is one-dimensional or spherical, respectively [26]. By assuming that the spot size is much larger than the penetration depth, a relatively planar heating and consequently 1-D heat transfer along the cross-plane direction is achieved. This constitutes a critical assumption for most optical-based measurements. Because temperature variation in optical-based measurements is too small to cause changes in the properties, material properties are assumed constant during the measurements.

\subsection{Heat transfer in a single-layer structure}

As the simplest case, we start with the heat transfer in single-layer materials with isotropic and temperature-independent thermal properties. Under such circumstances, the heat transfer is only onedimensional and has already been discussed in multiple resources [15-17, 21]. By adopting a periodic heat flux $q_{0} e^{-j \omega t}$ with amplitude $q_{0}$ and frequency $\omega$ as the boundary condition, the analytical solution that depicts 1-D heat transfer in a single-layer structure contains a phase shift of $\left(z / L_{p}+\pi / 4\right)[15-17,24,26]$, in which $L_{p}$ is the thermal penetration depth. It is seen from this expression that the phase shift is only dependent on thermal diffusivity, $\alpha$, based on the definition of thermal penetration depth. Therefore, the 
phase information is relatively robust compared with amplitude when determining the thermal properties of interest. As validation of the proposed heat transfer model, we performed PSTTR experiments on plain silicon substrates of different thicknesses and fit the experimental data with the solution to determine the thermal diffusivity.

\subsection{Heat transfer in multilayer structures}

In a separate publication by the authors [24], we presented a heat transfer model in a three-layer structure and derived the solution. Following the same geometry and analogous boundary conditions introduced in that work, a general heat transfer model in a multilayer structure (Fig. 1) is established. This multilayer structure has an arbitrary number of layers, denoted as " $n$." For a typical multilayer structure, as shown in Fig. 1, the governing equation is similar to the one- or three-layer structure:

$$
\frac{1}{\alpha_{i}} \frac{\partial T_{i}(r, z, t)}{\partial t}=\frac{1}{r} \frac{\partial}{\partial r}\left(r \frac{\partial^{2} T_{i}(r, z, t)}{\partial r}\right)+\frac{\partial^{2} T_{i}(r, z, t)}{\partial z^{2}}
$$

where $\alpha$ is the thermal diffusivity, $T_{i}$ is the temperature distribution in layer $i, t$ is time, $r$ is the radial direction coordinate, and $z$ is the coordinate along thickness direction.

After Laplace and Hankel transforms, the governing equation is changed into an ordinary differential equation along with the general solution in Eq. (2) to express the temperature distribution in the transformed domain. Boundary conditions also need to be transformed to determine the coefficients:

$$
w_{i}(\lambda, z, s)=A_{i} \cosh \left(\eta_{i} z\right)+B_{i} \sinh \left(\eta_{i} z\right)
$$

where $w_{i}$ is the temperature field in layer $i$ after Laplace and Henkel transforms, $A_{i}$ and $B_{i}$ are coefficients of the temperature solution, and $\eta$ is the transformed parameter. 


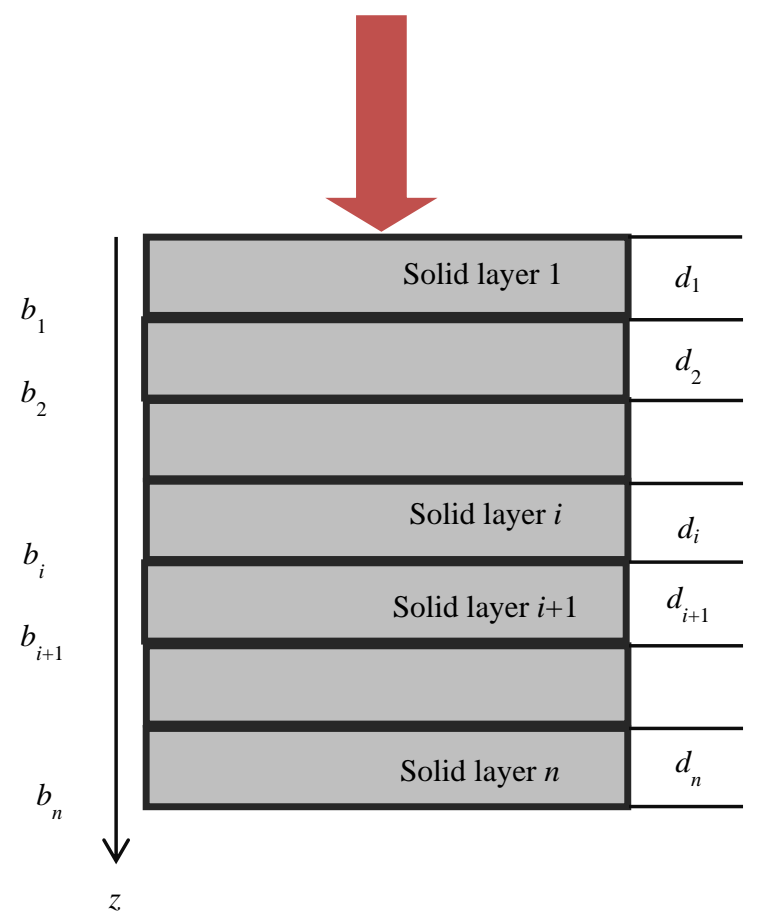

Fig. 1. Schematic of a general multilayer structure composed of $n$ layers.

At $b_{n}$, the boundary is assumed to be thermally insulated. After substituting Eq. (2) in Eq. (1), the insulated boundary condition is further simplified as:

$$
w_{n}=B_{n} \cdot \cosh \left[\eta_{n} \cdot\left(z-b_{n}\right)\right],
$$

from which it is seen that only one coefficient, $B_{n}$, is required to determine the temperature distribution on the bottom surface.

The top surface receives modulated heating, and the boundary condition after Hankel transform is also used to determine the coefficient $A_{1}$ and $B_{1}[16,24]$ :

$$
A_{1}=-\frac{q_{0}}{k_{1}} \frac{1}{(s+j \omega)} \cdot \frac{H(f(r))}{\eta_{1}}
$$

$B_{1}$ is determined to be 0 here. The function $f(r)$ is assumed to be a uniform function regardless of the pump beam's actual distribution. This assumption helps reduce another dimension along the radial 
direction. For all other layers in between, boundary conditions at each interface $(1,2, \ldots, n-1)$ are summarized as:

$$
\begin{gathered}
\left.k_{i} \frac{\partial w_{i}}{\partial z}\right|_{z=b_{i}}=\left.k_{i} \frac{\partial w_{i}}{\partial z}\right|_{z=b_{i+1}} ; \\
\left.k_{i} \frac{\partial w_{i}}{\partial z}\right|_{z=b_{i}}=\left.h_{i} \cdot\left(w_{i+1}-w_{i}\right)\right|_{z=b_{i}} .
\end{gathered}
$$

where $k_{i}$ is the thermal conductivity of layer $i$ and $h_{i}$ is thermal conductance between layers $i$ and $i+1$.

Substituting the general expression Eq. (2) into the boundary conditions at each interface, a recursive coefficient matrix is established as follows:

$$
\left(\begin{array}{l}
A_{i+1} \\
B_{i+1}
\end{array}\right)=D_{i} \cdot\left(\begin{array}{l}
A_{i} \\
B_{i}
\end{array}\right)
$$

in which $A_{i}, B_{i}, A_{i+1}$, and $B_{i+1}$ are coefficients in the solutions for the adjacent layers $i$ and $i+1 . D_{i}$ is a $2 \times 2$ matrix. Its elements are presented in Appendix A.

At $i=\mathrm{n}-1$, the matrix is directly derived following Eq. (6):

$$
\left(\begin{array}{l}
A_{n} \\
B_{n}
\end{array}\right)=D_{n-1} \cdot\left(\begin{array}{l}
A_{n-1} \\
B_{n-1}
\end{array}\right)
$$

where, because of the known geometrical relations, we define $S_{n,(n-1), n}=\sinh \left[\eta_{n} \cdot\left(b_{n-1}-b_{n}\right)\right]=$ $-\sinh \left(\eta_{n} \cdot d_{n}\right)=-S_{n n}$, and $C_{n,(n-1), n}=\cosh \left[\eta_{n} \cdot\left(b_{n-1}-b_{n}\right)\right]=\cosh \left(\eta_{n} \cdot d_{n}\right)=C_{n n}$. Thus, each element in the coefficient matrix is slightly different from the one associated with other interfaces. Details of the coefficient matrix $D_{n-1}$ are listed out in Appendix A.

With known interface coefficient matrices for all the interfaces, we can connect the coefficient set in the solution for an arbitrary layer $\left(A_{i}\right.$ and $\left.B_{i}\right)$ back to the coefficient set for the first layer $\left(A_{1}\right.$ and $\left.B_{1}\right)$, which were presented in Eq. (4):

$$
\left(\begin{array}{l}
A_{n} \\
B_{n}
\end{array}\right)=D_{n-1} \cdot\left(\begin{array}{l}
A_{n-1} \\
B_{n-1}
\end{array}\right)=D_{n-1} \cdot D_{n-2} \cdot\left(\begin{array}{l}
A_{n-2} \\
B_{n-2}
\end{array}\right)=D_{n-1} \cdot D_{n-2} \cdots D_{1}\left(\begin{array}{l}
A_{1} \\
B_{1}
\end{array}\right)
$$


For example, for the bottom surface, coefficient $A_{n}$ equals 0 and $B_{n}$ is derived as a function of $A_{1}$ $\left(A_{1}=-\frac{q_{0}}{k_{1}} \frac{1}{(s+j \omega)} \cdot \frac{\mathrm{H}(f(r))}{\eta_{1}}\right)$ :

$$
B_{n}=\mathcal{R}\left(D_{n-1} \cdot D_{n-2} \cdots D_{1}\right) \cdot A_{1}=-\mathcal{R}\left(D_{n-1} \cdot D_{n-2} \cdots D_{1}\right) \cdot \frac{q_{0}}{k_{1}} \frac{1}{(s+j \omega)} \cdot \frac{\mathrm{H}(f(r))}{\eta_{1}}
$$

The multiplied matrix denoted by $\mathcal{R}$ is solvable using either algebraic methods or more conveniently, using commercial mathematical software such as MATLAB. By substituting the coefficient $B_{n}$ back into Eq. (3), we finally solve for the transformed temperature distribution in the bottom layer. If the focus is only on the bottom surface $\left(z=b_{n}\right)$ and in the middle of heated area $(r=0)$, the temperature variation is further solved as:

$$
T\left(0, b_{n}, t\right)=\frac{q_{0} e^{-j \omega t}}{k_{1}} \int_{\lambda=0}^{\infty}\left\{-\mathcal{R}\left(D_{n-1} \cdot D_{n-2} \cdots D_{1}\right) \cdot \frac{\mathrm{H}(f(r))}{\eta_{1}}\right\}_{s=-i \omega} \lambda d \lambda
$$

Most previously reported work on heat transfer models stop here without simplification. In this work, in order to validate this model and also utilize the solution for practical cases, we completed the integral by assigning the number of layers " $n$ " several specific values, such as 1,2 , and 3 , to derive the corresponding temperature distributions. When the value of $n$ is relatively small ( 2 or 3 ), it is easy to calculate the coefficient matrices directly and implement the integral. The corresponding temperature distribution derived using Eq. (10) when $n$ equals 2 and 3 are identical to those found in other publications $[16,24]$. More details about the validation process are presented in Appendix B. Next, we discuss the physical significance behind the analytical solutions that represent the temperature variation on the bottom surface for the two-layer and the three-layer structures. When the optical signal reaches the heating surface, it is firstly absorbed by the energy carriers within the heating spot area, and this localized area quickly achieves a uniform temperature increase. Then, the excited energy carriers interact with surrounding energy carriers and transfer the energy to them. Depending on the ratio of the spot size and the penetration depth, this heating is either planar or spherical [26]. In this work, we have ensured that the spot size is considerably greater than the penetration depth. Therefore, the heat source is planar, and the 
phase shift between the source and the instantaneous temperature response at the heating spot is $\pi / 4$, which is explicitly observed in the solutions. After the surface temperature rises, the subsequent thermal wave penetrates into the material and this traveling thermal wave is expressed by $\left(d / L_{p}\right)$. The traveling thermal wave indicates that the bulk material in each layer also affects the phase shift to a certain extent. In the inverse tangential term, we explicitly observe contributions from the interfaces in the form of the contact resistance $R$ and thermal effusivity [27], defined as $\sqrt{k \rho c_{p}}$. Thermal effusivity essentially measures the material's ability to exchange the heat with its surroundings, and it usually has a depth of a few micrometers in the region adjacent to the interface. Unlike the serial influences on the traveling thermal wave from bulk material layers, the influence from the interfaces, including the contact resistance and effusivity, are highly nonlinear and coupled in the inverse tangential component. To conduct a more in-depth study on the contributions to the phase shift from the interfaces, we select the solution for a twolayer structure $[16,24]$ for further derivation. Given the definitions $k=\alpha \cdot \rho \cdot c$ and $L_{p}=\sqrt{2 \alpha / \omega}=$ $\sqrt{\alpha / \pi f}$, the phase shift through a two-layer structure becomes:

$$
\Phi=\frac{\pi}{4}+d_{1} \sqrt{\frac{\pi f}{\alpha_{1}}}+d_{2} \sqrt{\frac{\pi f}{\alpha_{2}}}+\tan ^{-1}\left[\frac{\left(\sqrt{\pi f(k \rho c)_{2}} \cdot R_{12}\right)}{\left(1+\sqrt{\frac{(k \rho c)_{2}}{(k \rho c)_{1}}}+\sqrt{\pi f(k \rho c)_{2}} \cdot R_{12}\right)}\right]=\frac{\pi}{4}+d_{1} \sqrt{\frac{\pi f}{\alpha_{1}}}+d_{2} \sqrt{\frac{\pi f}{\alpha_{2}}}+\tan ^{-1}\left[\frac{\frac{R_{12}}{R_{L_{p}, 2}+R_{L_{p}, 1}}}{1+\frac{R_{12}}{R_{L_{p}, 2}+R_{L_{p}, 1}}}\right]
$$

where, similar to the definition of thermal resistance $(R=L / k)$, we define two equivalent resistances, $R_{L p, 1}$, and $R_{L \mathrm{p}, 2}$. Analogous to the conventional definition of thermal resistance as length divided by the thermal conductivity, this equivalent thermal resistance is defined using the thermal penetration depth and represents the resistance from a thermal path that only equals the penetration depth. The ratio $\frac{R_{12}}{R_{L_{p}, 2}+R_{L_{p}, 1}}$ indicates a comparison between the interface contact resistance and summation of the equivalent resistances from the bulk materials adjacent to the interface. These two resistance parameters determine how the phase is affected when thermal energy travels through the interface. From Eq. (11), the impact from the interfaces is expressed by ratios of parameters rather than discrete parameters. It is understandable because when thermal energy travels through the interface, a portion of it gets reflected by 
the interface while the rest goes through the interface. Therefore, the reflected thermal energy is a form of secondary heat transfer that penetrates backward and causes secondary effects on the phase shift. With Eq. (11), the interfacial effect on the heat transfer becomes clearer analytically. If the frequency increases, the penetration depth in the materials gets reduced, and therefore the equivalent thermal resistance decreases. Then the resistance from the surface contact becomes relatively greater, leading to a larger phase shift. With the same materials/layers and the same frequency range, a larger contact resistance leads to a larger phase shift. On the other hand, with the contact resistance being the same, a material with higher bulk thermal conductivity contributes to a smaller thermal resistance than the contact resistance as compared to a material with a lower bulk thermal conductivity. Therefore, the ratio between the contact resistance and equivalent resistances increases, leading to a larger phase shift. Fig. 2 depicts how the phase shift associated with the inverse tangential term varies with different contact resistances and different materials over a frequency range of 100 to $10^{4} \mathrm{~Hz}$ in a two-layer structure, which is usually used for studying micrometer-scale materials/structures. In Fig. 2(a), four different types of materials are simulated—silicon, copper, aluminum, and silicon dioxide - under an identical contact resistance of $1 \mathrm{~mm}^{2} \cdot \mathrm{K} / \mathrm{W}$ between the two substrates of the same materials. From Eq. (11), the ratio between contact resistance and equivalent thermal resistances from penetration depth in adjacent layers together determine the overall phase shift. Higher bulk thermal conductivity reduces the equivalent thermal resistance, so the contact resistance imposes greater influence on the overall phase shift. In Fig. 2(b), we select silicon as the substrate and vary the contact resistance between the two silicon substrates from 0.01 to $10 \mathrm{~mm}^{2} \cdot \mathrm{K} / \mathrm{W}$. If the contact resistance is increased, the ratio between contact resistance and equivalent resistance increases. Thus, the phase shift from interface transport also increases. The simplification and explicit algebraic relationship in Eq. (11) motivates us to explore a general analytical solution for phase shift in structures with more than two layers using a similar equivalent resistance definition for each interface. Even though the inverse tangential term is complicated, a pattern seemingly exists, and it may be instructive to derive the analytical phase shift without going through all the mathematical derivations. To achieve this goal, we 
define a lumped parameter, $\mathcal{R} \mathcal{R}_{i, i+1}=\frac{R_{i, i+1}}{R_{L_{p}, i}+R_{L_{p}, i+1}}$, to avoid presenting a large number of ratio terms when treating cases with more than three layers. Thus, a general solution for the phase shift on the bottom side of an $n$-layered structure is arrived at as follows:

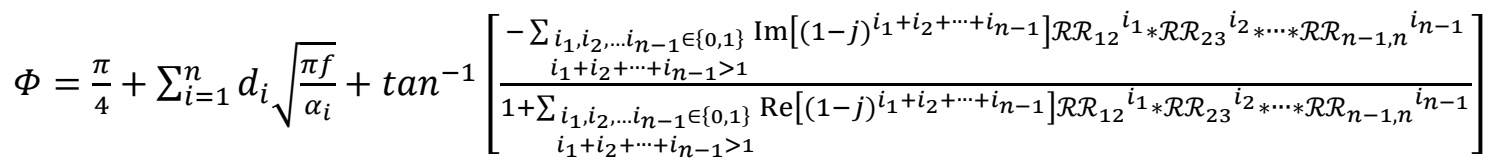

in which the parameter $\mathcal{R} \mathcal{R}_{i, i+1}=\frac{R_{i, i+1}}{R_{L_{p}, i}+R_{L p, i+1}}$ is calculated with each interface; therefore, there are $n-1$ terms. The definition of this lumped parameter presents substantial advantages over other models and solutions when dealing with the multilayer structures. First, the variable $R_{L p}$ associated with each layer is calculated individually and is then substituted into the lumped parameter $\mathcal{R} \mathcal{R}$, along with the contact resistance $R$. Second, the calculation of Eq. (12) only involves summations of multiple terms and is easily achieved using the loop structure programming or commercial software such as MATLAB. Here, we need to explain the meaning of the two summation terms in Eq. (12) to help better implementation of this equation. These two summation terms in Eq. 12 are defined based on the theory of combination, by choosing $i$ elements from the set of $n-1$ elements, $\mathcal{R} \mathcal{R}_{12}, \mathcal{R} \mathcal{R}_{23}, \ldots, \mathcal{R} \mathcal{R}_{n-1, n}$, and then multiplying the $i$ elements together. The total number of selected elements evolves from 1 to $n$-1. For example, if $n$ equals 2, there is only one term, $\mathcal{R} \mathcal{R}_{12}=\frac{R_{1,2}}{R_{L_{p, 1}}+R_{L_{p}, 2}}$, and Eq. (12) is reduced into an expression as reported in [16]; if $n$ equals 3 , it becomes $(1-j) \frac{R_{1,2}}{R_{L p, 1}+R_{L p, 2}}+(1-j) \frac{R_{2,3}}{R_{L p, 2}+R_{L p, 3}}+(1-j)^{2} \frac{R_{1,2}}{R_{L p, 1}+R_{L p, 2}} \cdot \frac{R_{2,3}}{R_{L p, 2}+R_{L p, 3}}$ and is in exactly the same form as the expression from other work [16, 24]. If $n$ is greater than 3 , this term will contains many more combinations, therefore making Eq. (12) complicated. $\operatorname{Re}(1-j)^{i}$ and $\operatorname{Im}(1-j)^{i}$ denote the real part and the imaginary part of $(1-j)^{i}$, respectively. While it is feasible to write out all the combinations for small $n$, MATLAB is a more effective alternative method to calculate all combinations when $n$ is larger than 3. Other than the obvious mathematical simplicity, Eq. (12) allows for the fundamental interpretation of the thermal energy transport in multilayer structures. Although the 
propagation of the thermal wave inside materials/layers has been studied and reported, due to its complex nature and accompanying mathematical transforms, the analytical solutions usually show certain levels of complications, such as integrals and exponential terms. These complications prevent application of the solutions in practical cases to a significant extent. If the temperature distribution is solved using numerical methods, the numerical solutions are incapable of revealing the fundamental physics behind the phenomena. Compared with other optical-based techniques that study the phase signal as well, Eq. (12) offers a clearer interpretation of the thermal energy transport within multilayer structures. It indicates that when thermal energy propagates inside materials with interfaces, these interfaces create multiple resistances to the heat transfer because of the reflection and transmission of thermal energy at each interface. The resistances caused by these multiple reflections and transmissions are consequently cumulative, coupled, and non-linear, as seen in Eq. (12). The overall phase shift is thus complicated when considering the cumulative effects from the traveling wave and the interface reflection/transmission, along with the resonance effects from the bottom surface. The explicit expression in Eq. (12) not only enables researchers to acquire the analytical solution, but also helps them to comprehend the contribution from different layers. With Eq. (12), if the physical parameters are known, it is easy to calculate the phase shift directly without performing complicated derivations. Given that most multilayer structures usually contain two, three, or four layers, it is straightforward to apply Eq. (12) and see which layers have the most significant influence on the heat transfer. 

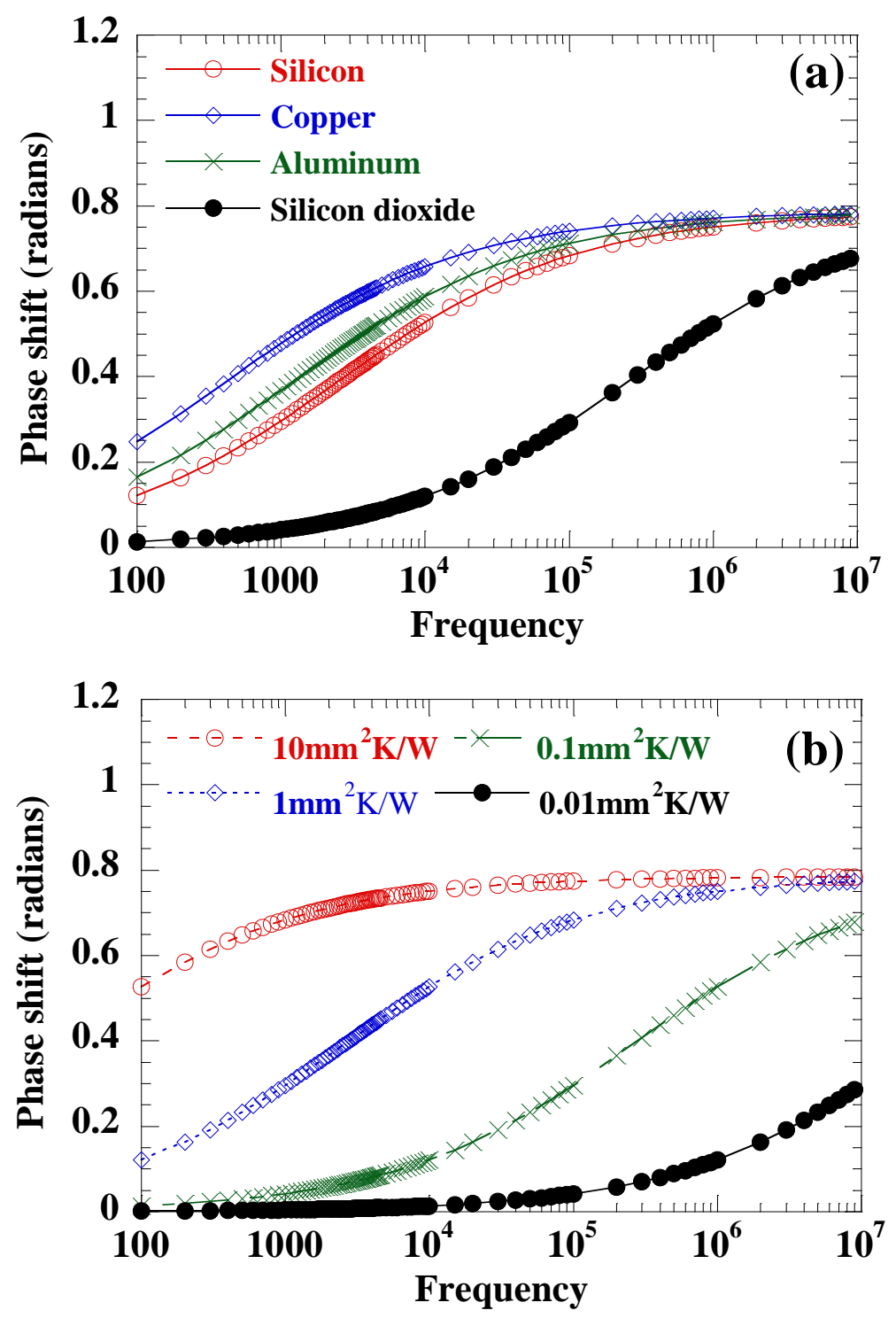

Fig. 2. (a) Phase shifts caused by thermal energy transport through interfaces for different substrate materials (silicon, copper, aluminum, and silicon dioxide) over a frequency range from 100 to $10^{7} \mathrm{~Hz}$. The contact resistance is set to be $1 \mathrm{~mm}^{2} \cdot \mathrm{K} / \mathrm{W}$; (b) Phase shifts caused by thermal energy transport through interfaces of different contact resistances, over a frequency range from 100 to $10^{7} \mathrm{~Hz}$. The substrate material is silicon. 


\section{EXPERIMENTAL APPROACH}

\subsection{Experiment setup of PSTTR}

We now turn to the application of the heat transfer model presented in the previous section to determine thermal properties of materials using the PSTTR technique. A schematic of the experimental setup is shown in Fig. 3. Its principle was introduced in a different publication by the authors [24]. Among the factors that affect the measurements, the alignment of the two laser signals is the most critical, as it strongly influences the strength of the signal into the photodiode [16]. The pump laser was mounted on a motorized stage, ensuring accurate adjustment of the laser spot. The probe laser spot was first fixed and marked using a charge-coupled device camera. The pump laser was then fine tuned using the motorized stages to identify the best alignment by observing the intensity readings of the lock-in amplifier. This alignment process was usually delicate and difficult due to the small but oscillating temperature response on the back surface. In addition, the appropriate ratio between the two spot sizes was important for the success of the experiment.

Based on a comparison between the photodiode signal and the reference input of the modulation frequency, the phase difference extracted by the lock-in amplifier represented a backward shift from the original periodic waveform. This phase shift was not only composed of the effect of the thermal transport in the materials, but also was affected by the devices and equipment used in the experiments. Therefore, we conducted a calibration to quantitatively determine the systematic and environmental effects. For this purpose, the experiment was first run at the same frequency series without mounting the sample, and the phase data were recorded as a baseline. This baseline phase shift was subtracted from the phase shift obtained when the samples were included in the experiment. This systematic phase shift was usually consistent at one value despite changes in the laser parameters. 


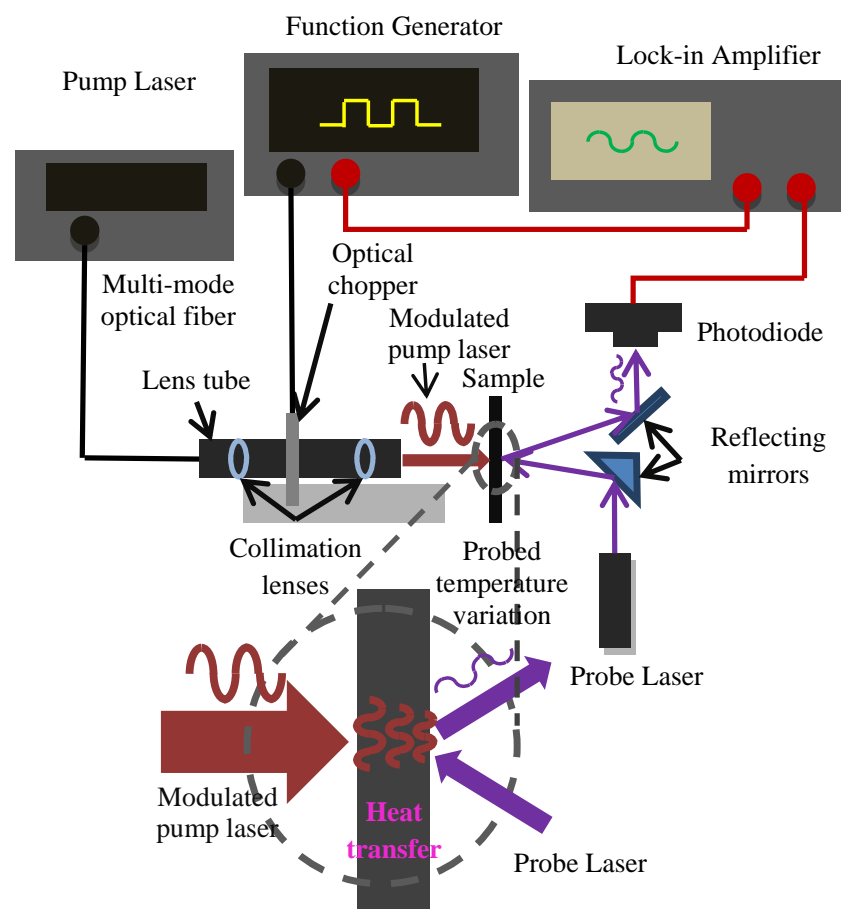

Fig. 3. Experimental configuration of the frequency-domain transient thermoreflectance technique used to characterize the TIMs/BIMs.

\subsection{Sample preparation}

To validate the multilayer heat transfer model using PSTTR, we selected a variety of test articles for experimentation. Plain silicon substrates of various thicknesses were chosen to be the baseline test article for the single-layer model. Silicon has well-documented physical/thermal properties and is also commonly used in the semiconductor industry. Both sides of the silicon substrates were cleaned and polished to have mirror-like reflective features. To enhance the confidence in our validation, we characterized silicon substrates of various thicknesses ranging from $100 \mu \mathrm{m}$ to $280 \mu \mathrm{m}$. We controlled the frequency to vary from $10^{3} \mathrm{~Hz}$ to $10^{4} \mathrm{~Hz}$, and the thermal penetration depth was calculated to change from $150 \mu \mathrm{m}$ to $50 \mu \mathrm{m}$. Therefore, we successfully maintained the penetration depth to be much lower than the thickness, thereby satisfying the assumption of "thermally thick samples." 
To apply the analytical models of the multilayer structures, we prepared both three-layer and fourlayer samples. For the three-layer sample, we utilized commercially available thermal grease (Dow Corning TC-5022) as the interface layer and silicon substrates of $100-\mu \mathrm{m}$ thickness as the bounding substrates. After cleaning the surface, a small quantity of TC-5022 was applied to one substrate, and the second substrate was pressed onto the grease and squeezed to cause the grease to flow between the two substrates. The samples were further annealed under elevated temperature and under pressure to create the thinnest possible layer of grease. A grease layer thickness of 60 to $70 \mu \mathrm{m}$ was achieved, which is a typical bond line thickness of greases. This sample represents a standard commercial method of creating thermal contact between cold plates and electronic components.

For the four-layer sample provided by Delphi, two silicon substrates were directly bonded under elevated temperature and pressure. Both silicon substrates were pre-coated with a thin aluminum layer of about $2-\mu \mathrm{m}$ thickness to assist the bonding. The two aluminum surfaces were brought into proximity at room temperature and pressed with a small amount of force to initiate the bonding, following by thermal annealing at $550^{\circ} \mathrm{C}$ and $3 \mathrm{MPa}$ for 3 hours. The overall thickness of the fabricated structure was about $1,200 \mu \mathrm{m}$, which was then polished down to $200 \mu \mathrm{m}$ with a chemical-mechanical planarization process. Due to the small thickness and high thermal conductivity of the aluminum layers, the multilayer samples could be assumed to contain only two layers. However, to be rigorous and to potentially extract interesting information about the various contact resistances, we still treat the direct-bonded assembly as a four-layer sample. A microscopic image of the bond line is shown in Fig. 4, with the thickness marked for the two silicon substrates. 


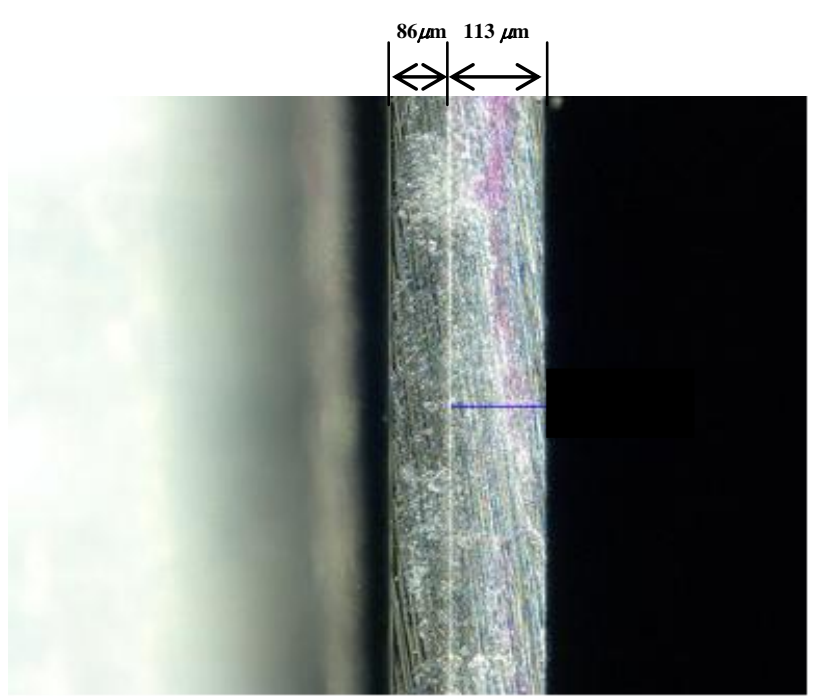

Fig. 4. Microscopic side view of the direct-bonded sample, showing the thicknesses of the two bonded silicon substrates (credit: Charlie King, NREL). 


\section{RESULTS AND DISCUSSIONS}

\subsection{Single-layer structure}

To validate the proposed heat transfer model for multilayer structures, three test articles containing different numbers of layers were prepared, as introduced in section 3.2. We first conducted experiments on single silicon substrates because the properties of silicon have been studied extensively and are well known. The test results from the silicon substrates established a reliable baseline for all subsequent tests on other samples. Silicon substrates of different thicknesses $(100,170$, and $280 \mu \mathrm{m})$ were tested using the PSTTR technique. Typical phase shift-frequency data points are plotted in Fig. 5. For comparison, a curve from the theoretical heat transfer analysis is also added (solid red dots and line). As shown by the analytical solution, in the single-layer structure the phase shift is linearly dependent on the term $b / L_{p}$, which is defined as the normalized frequency and has an intercept of $-\pi / 4$. In Fig. 5, when the normalized frequency $b / L_{p}$ is chosen as the $x$ axis, the experimentally measured data points from the silicon substrates of various thicknesses all converge close to the theoretical line. Only when the material is "thermally thick" is the total phase shift quantified as $\left(\pi / 4+b / L_{p}\right)[17]$. Therefore, we selected a frequency range that assured that the thickness of the sample/substrate was larger than the penetration depth. Usually the ratio $b / L_{p}$ needs to be greater than 0.8 , which consequently defines the low-frequency limit. In Fig. 5 , the data in the region of $b / L_{p}>1.0$ are intentionally chosen for global fitting. For instance, for a silicon substrate of $100-\mu \mathrm{m}$ thickness, the thermal diffusivity is fitted to be about $7.9 \times 10^{-5} \mathrm{~m}^{2} / \mathrm{s}$. The density and specific heat of silicon are $2,230 \mathrm{~kg} / \mathrm{m}^{3}$ and $712 \mathrm{~J} /(\mathrm{kg} \cdot \mathrm{K})$, respectively [28]. The thermal conductivity is calculated to be $131 \mathrm{~W} /(\mathrm{m} \cdot \mathrm{K})$. For silicon substrates as thin as $100 \mu \mathrm{m}$, due to the size effects, most phonons have a mean free path (MFP) between $10 \mathrm{~nm}$ to $1 \mu \mathrm{m}$ at room temperature. Henry and Chen's simulation shows that at $300 \mathrm{~K}$, phonons with MFPs between $100 \mathrm{~nm}$ to $10 \mu \mathrm{m}$ comprise $\sim 70 \%$ of the thermal conductivity, while the phonons with MFPs larger than $100 \mu \mathrm{m}$ only account for less than $10 \%$ contribution to the thermal conductivity [29]. Therefore, the anticipated thermal conductivity for the silicon substrates tested in this work would be lower than the documented bulk value of approximately $148 \mathrm{~W} /(\mathrm{m} \cdot \mathrm{K})$ [28]. Due to 
the lack of necessary information about the grain structure and doping information for the silicon substrates, it is hard to estimate the thermal conductivity, but the statistical results of $131 \mathrm{~W} /(\mathrm{m} \cdot \mathrm{K})$ fall within the reasonable range. When the thickness varies, the derived thermal diffusivity does not show a significant change because the normalized frequency-phase curves at different thicknesses converge to the theoretical curve. The parameters adopted in the fitting procedures and the results, along with the uncertainties are listed in Table 1.

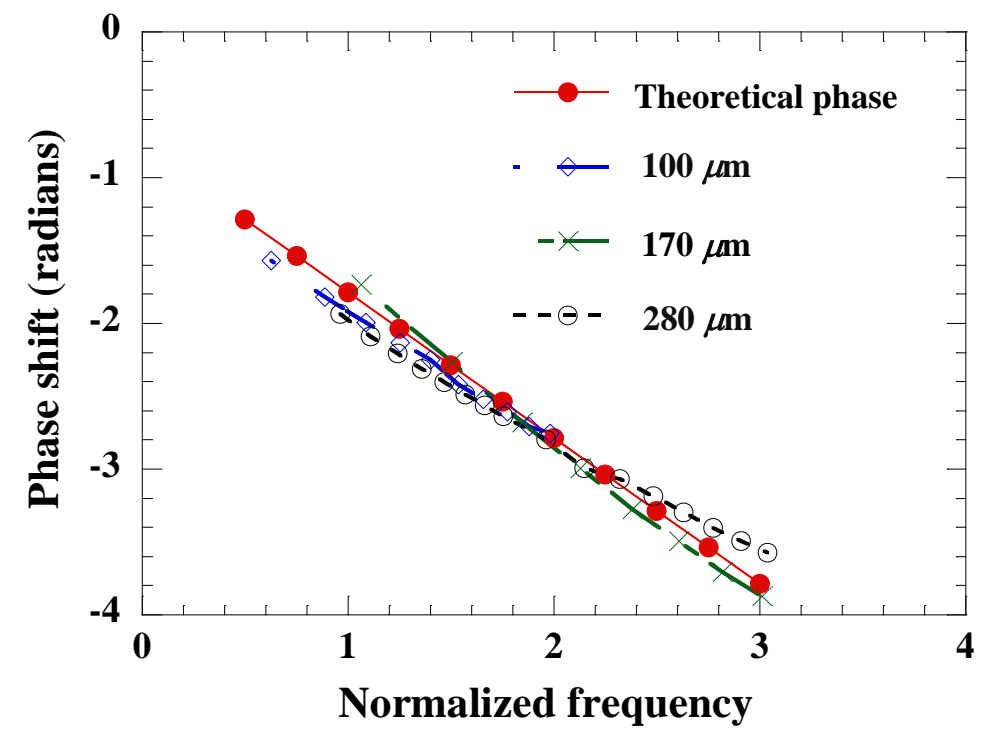

Fig. 5. Experimental phase shift as a function of normalized frequency for plain silicon substrates of different thicknesses. The theoretical phase shift profile (red dots) is added for comparison purposes. 


\section{Table 1}

Parameters and results for two different materials (silicon substrate and thermal grease TC-5022) measured using the PSTTR technique.

\begin{tabular}{c|c|c}
\hline Parameters & Silicon substrate & TC-5022 \\
\hline Total Thickness $(\mu \mathrm{m})$ & 100 & 270 \\
\hline Bond line thickness $(\mu \mathrm{m})$ & & 70 \\
\hline Density $\left(\mathrm{kg} / \mathrm{m}^{3}\right)$ & 2,330 & 3,230 \\
\hline Specific heat $(\mathrm{J} /(\mathrm{kg} \cdot \mathrm{K}))$ & 712 & 251 \\
\hline Bulk thermal conductivity $(\mathrm{W} /(\mathrm{m} \cdot \mathrm{K}))$ & $131 \pm 6 \%$ & $3.5 \pm 22 \%$ \\
\hline Thermal diffusivity $\left(\mathrm{m}^{2} / \mathrm{s}\right)$ & $7.9 \times 10^{-5} \pm 6 \%$ & $4.3 \times 10^{-6} \pm 22 \%$ \\
\hline Contact resistance $\left(\mathrm{mm}^{2} \cdot \mathrm{K} / \mathrm{W}\right)$ & & $8.7 \pm 16 \%$ \\
\hline Total thermal resistance $\left(\mathrm{mm}^{2} \cdot \mathrm{K} / \mathrm{W}\right)$ & & $37.1 \pm 27 \%$ \\
\hline
\end{tabular}

\subsection{Multi-layer structures}

Results from single-layer silicon substrates have established a solid validity of this model and the characterization of multi-layer (three-layer and four-layer) structures using the model would substantially show its capability on complicated structures. The three-layer structure was fabricated by sandwiching thermal grease in between two plain silicon substrates, as introduced in the prior section: there are two substrates and one interface layer between the two substrates. The geometrical, physical, and thermophysical properties of each layer were recorded and used in the three-layer heat transfer analysis. The overall thickness of the grease layer was slightly different from spot to spot, varying from 60 to 70 $\mu \mathrm{m}$. As analyzed above, Eq. (12) gives the phase shift in the high-frequency region by setting the number of layers to be three. In addition, the analytical solution of the temperature distribution in the three-layer structure suggests that the amplitude of temperature oscillation on the opposite surface exponentially decreases when the frequency increases. On the other hand, if the modulation frequency decreases, the penetration depth increases, which may violate both the "1-D" and "thermally thick" assumptions. Thus, based on the heat transfer modeling, to both assure a signal strong enough to suppress the environmental 
disturbance and satisfy the assumption of a 1-D heat transfer in a thermally thick material, the frequency range was chosen to be between $1 \mathrm{kHz}$ and $3 \mathrm{kHz}$. In Fig. 6, typical phase shift data for the three-layer structure are plotted against the normalized frequency. A profile based on the theoretical modeling is added for comparison. The data points show minor oscillation and are very close to the theoretical profile. Because the PSTTR technique considers overall interfacial thermal resistance as a combined effect from discrete components, there are several undetermined interface material properties, such as density, specific heat, thickness, and thermal diffusivity, as indicated in Eq. (12). Some properties are documented in the literature and used as constants through the fitting process, while some other properties, such as thermal conductivity, thermal diffusivity, and contact resistance, remain unknown. Theoretical solutions from the heat transfer analysis were adopted in the fitting process to identify the best value sets. To conduct data fitting, initial values for the variables to be fitted are needed. Depending on the nature of the convergence, the initial values could be random numbers or reasonable guesses. In the three-layer structures, we selected TC-5022 as the material with unknown properties. Fig. 6 shows a typical fitting graph to obtain the best fit parameters. To eliminate the random uncertainty with a particular spot, we moved the sample around so that the lasers probed different spots. For the experimental data obtained at each spot, we conducted the data fitting process to acquire a set of values. The average thermal diffusivity was determined to be $4.3 \times 10^{-6} \mathrm{~m}^{2} / \mathrm{s}$, and the contact resistance of the silicon-TC-5022 interface was 8.7 $\mathrm{mm}^{2} \cdot \mathrm{K} / \mathrm{W}$, which represents the single interfacial/contact thermal resistance only. TC-5022's density is $3,230 \mathrm{~kg} / \mathrm{m}^{3}$ [30], and its specific heat was measured to be $251 \mathrm{~J} /(\mathrm{kg} \cdot \mathrm{K})$, using a differential scanning calorimetry. Thus, the average thermal conductivity of TC-5022 is calculated to be $3.5 \mathrm{~W} /(\mathrm{m} \cdot \mathrm{K})$, close to the reported value of $4.0 \mathrm{~W} /(\mathrm{m} \cdot \mathrm{K})$ in the data sheets as well as measurements via the ASTM technique [31]. With the fitted thermal conductivity and contact resistance, the overall thermal resistance of the grease layer is $37.1 \mathrm{~mm}^{2} \cdot \mathrm{K} / \mathrm{W}$. From the data fitting, we derived the resistance from one surface contact as $8.7 \mathrm{~mm}^{2} \cdot \mathrm{K} / \mathrm{W}$. Therefore, the overall resistance from surface contacts (i.e., contact resistance) is 17.4 $\mathrm{mm}^{2} \cdot \mathrm{K} / \mathrm{W}$ because of the structural symmetry. It is then concluded that almost $50 \%$ of the thermal resistance is caused by thermal transport within the material, and the other $50 \%$ is from the contact 
resistances. These insights into the interface layer reveal that the thermal grease material and contact surfaces have almost equal resistance to the heat flow. The average values of the fitted unknown parameters of TC-5022 and uncertainties are included in Table 1, with associated 95\% confidence level.

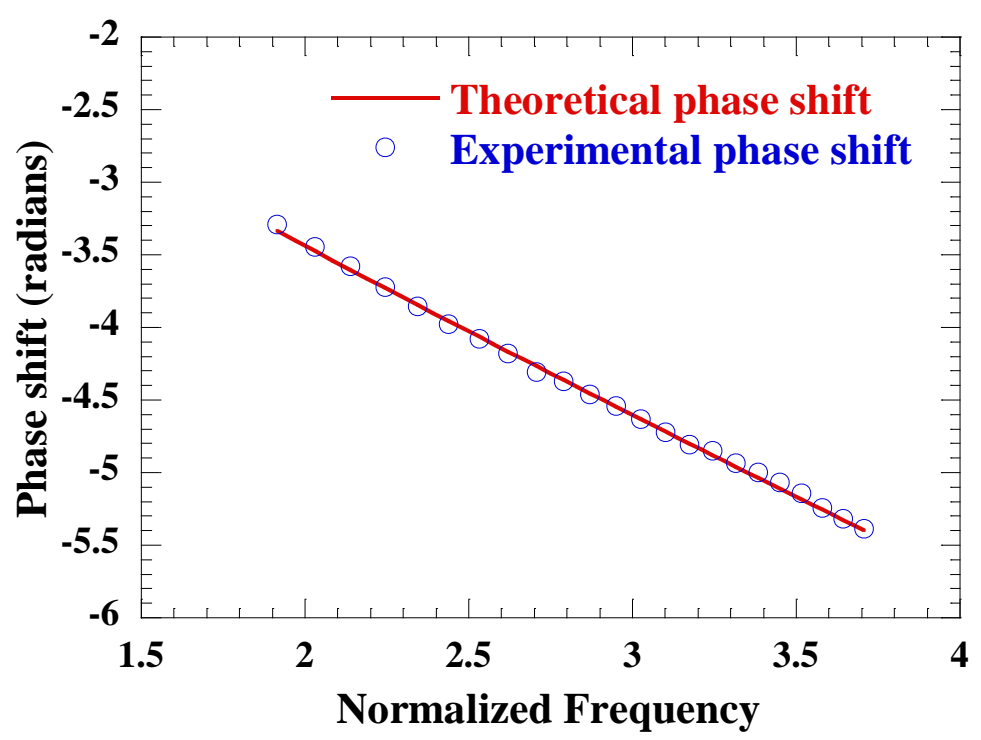

Fig. 6. Experimental and theoretical phase shift as a function of normalized frequency for the three-layer structure (silicon-grease-silicon).

The four-layer structure, which was provided by Delphi, was fabricated using the direct bonding technique to bond two pre-processed silicon substrates. Because direct bonding is based on atomic interactions, the quality is anticipated to be superior to conventional bonding materials. Based on the sample preparation process introduced in the prior section, this test article was considered to have four layers of material (silicon, aluminum, aluminum, silicon) and have three contact interfaces ( $\mathrm{Si}-\mathrm{Al}, \mathrm{Al}-\mathrm{Al}$, $\mathrm{Al}-\mathrm{Si}$ ). The general solution of the phase shift in Eq. (12) was used to analyze the measurement results. Similar to the three-layer heat transfer model, a constant $\pi / 4$ term and phase shifts from the thermal wave traveling in each layer remain the same. However, the multiple interfaces bring greater complexity into the solution. We calculated the resistance term $\frac{R_{i, i+1}}{R_{L_{p}, i}+R_{L p, i+1}}$ for each interface with both known and reasonable guesses for unknown parameters and then substituted them back into Eq. (12). The fitting 
graph for the four-layer direct-bonded sample is similar to the one for the three-layer structure shown in Fig. 6. Based on repeating the fitting process with multiple sets of data, the average overall thermal resistance was determined to be $0.46 \mathrm{~mm}^{2} \cdot \mathrm{K} / \mathrm{W}$, which includes the bulk resistances from the aluminum layers and the contact resistances from the $\mathrm{Si}-\mathrm{Al}$ and $\mathrm{Al}-\mathrm{Al}$ interfaces. Specifically, the contact resistance between silicon and aluminum surfaces is about $0.06 \mathrm{~mm}^{2} \cdot \mathrm{K} / \mathrm{W}$. According to work reported on solid interfaces, room-temperature contact resistances between metals and dielectrics should be about 0.01 to $0.1 \mathrm{~mm}^{2} \cdot \mathrm{K} / \mathrm{W}[32,33]$. In addition, the thermal resistance of the $2-\mu \mathrm{m}$ aluminum coating is less than 0.01 $\mathrm{mm}^{2} \cdot \mathrm{K} / \mathrm{W}$, which is negligible compared to that of other layers. The $\mathrm{Al}-\mathrm{Al}$ interface contact resistance is calculated to be $0.33 \mathrm{~mm}^{2} \cdot \mathrm{K} / \mathrm{W}$, meaning that over $70 \%$ of the thermal resistance is caused by this interface. The contact resistance is probably caused by phonon/electron scattering at this interface and also structural imperfections, such as voids and dislocations in the thin aluminum layers and adjacent silicon substrate. Because the aluminum layer is sputter-coated, the adhesion between silicon and aluminum is assumed to be optimal. In comparison, when the two aluminum surfaces are brought into contact at elevated temperature and additional pressure, the contact and consequent bonds may not be as good as that resulting from the sputter coating. Therefore, the $\mathrm{Al}-\mathrm{Al}$ interface induces stronger resistance to the heat flow. Similar to the PSTTR experiments with the three-layer structures, the four-layer sample was moved around so that multiple spots were measured. The averaged values for all fitted results are presented in Table 2 with associated uncertainties at 95\% confidence level. The total interfacial thermal resistance of the direct-bonded silicon wafers is impressively low compared with the thermal resistances in Table 1. 
Table 2

Parameters and results of direct-bonded sample measured using PSTTR technique and four-layer model.

\begin{tabular}{c|c}
\hline Parameters & Value \\
\hline Total thickness $(\mu \mathrm{m})$ & 200 \\
\hline Bond line thickness $(\mu \mathrm{m})$ & 4.00 \\
\hline Density of aluminum $\left(\mathrm{kg} / \mathrm{m}^{3}\right)$ & $2,702[28]$ \\
\hline Specific heat of aluminum $(\mathrm{J} /(\mathrm{kg} \cdot \mathrm{K}))$ & $903[28]$ \\
\hline Bulk thermal conductivity of aluminum $(\mathrm{W} /(\mathrm{m} \cdot \mathrm{K}))$ & $237[28]$ \\
\hline Thermal diffusivity of aluminum $\left(\times 10^{-5} \mathrm{~m}^{2} / \mathrm{s}\right)$ & $9.70[28]$ \\
\hline $\mathrm{Al}-\mathrm{Si}$ contact resistance $\left(\mathrm{mm}^{2} \cdot \mathrm{K} / \mathrm{W}\right)$ & $0.06 \pm 16 \%$ \\
\hline $\mathrm{Al}-\mathrm{Al}$ contact resistance $\left(\mathrm{mm}^{2} \cdot \mathrm{K} / \mathrm{W}\right)$ & $0.33 \pm 23 \%$ \\
\hline Overall thermal resistance $\left(\mathrm{mm}^{2} \cdot \mathrm{K} / \mathrm{W}\right)$ & $0.46 \pm 15 \%$ \\
\hline
\end{tabular}

\subsection{Measurement uncertainty and sensitivity}

To implement the PSTTR measurement, an automatic data acquisition system was built to collect the amplitude and phase extracted from the lock-in amplifier. For each frequency generated from the function generator, the sampling time was 60 seconds and the sampling rate was $10 \mathrm{~Hz}$. Then, the average value of 600 samples that were collected was recorded as the amplitude and phase associated with this frequency. This data acquisition system is estimated to have about 5 degrees random uncertainty. The lock-in amplifier has a systematic uncertainty of about 1 degree. Another critical source of error is from the regression analysis process. When fitting the theoretical solution to the experimental data, the discrepancy is minimized to achieve the best fit and determine the parameters of interest. An error comes in the fitting process, while the other modeling parameters, known or unknown, are used with different levels of uncertainty. Here, we adopted a methodology from Malen et al.'s work [26]. The uncertainty of the parameters to be determined is estimated from the uncertainties in the modeling parameters. After determining the best fit, each of the modeling parameters is perturbed by a small quantity (its uncertainty), and the regression process is repeated to find the new fitted value of the unknowns to be determined. 
Then, the difference between the new and old values is the uncertainty from the fitting process to determine the unknown parameters. Using this methodology and combining fitting uncertainty with the measurement uncertainty (random and systematic uncertainty), the overall uncertainty at $95 \%$ confidence level is calculated, as shown in Tables 1 and 2 .

To investigate the credibility of the experimental results, a sensitivity study of the output signal to the different parameters was conducted. We applied the analytical solution (Eq. (12)) to directly derive the analytical expression of the sensitivity to the various parameters. A differential definition of sensitivity was adopted from the work of Hopkins et al. [34] and is defined as:

$$
\left|S_{p}\right|=\left|\frac{\partial \ln \Phi}{\partial \ln p}\right|=\left|\frac{p}{\Phi} \cdot \frac{\partial \Phi}{\partial p}\right|
$$

where $\Phi$ is the phase shift and $p$ is any property of interest, such as $k$, the thermal conductivity, and $R$ the thermal resistance. This definition highlights another advantage associated with the analytical solution in Eq. (12). Compared with other numerical results or complicated mathematical solutions with multiple integrals, Eq. (12) is more straightforward to obtain differentiation with respect to particular parameters using the definition in Eq. (13). If we understand how the sensitivity qualitatively changes with the frequency, we can determine the optimal frequency to achieve more accurate measurements and which fitted parameter is more trustworthy. In Fig. 7, we plot the sensitivity to the thermal conductivity of each layer and the contact resistance of each interface versus frequency for the TC-5022 grease material. It is seen that the sensitivities to both the top and bottom silicon substrates are identical and so are the sensitivities to the two interfaces. The reason for this is that the multilayer structure is symmetric in the thickness direction. The phase signal shows stronger sensitivity to the silicon substrate than to the grease layer. It is observed that when the modulation frequency increases, the phase shift is more sensitive to the bulk thermal conductivity than to the contact resistance. However, in the low-frequency range, the phase shift is more sensitive to the thermal contact resistance. Therefore, other than the appropriate frequency range $(1 \mathrm{kHz}-3 \mathrm{kHz})$ based on heat transfer modeling, as stated in section 4.2 , the sensitivity analysis is 
also instructive for selecting the optimal frequency range $(500 \mathrm{~Hz}$ to $3 \mathrm{KHz})$ for optimally determining the property of interest.

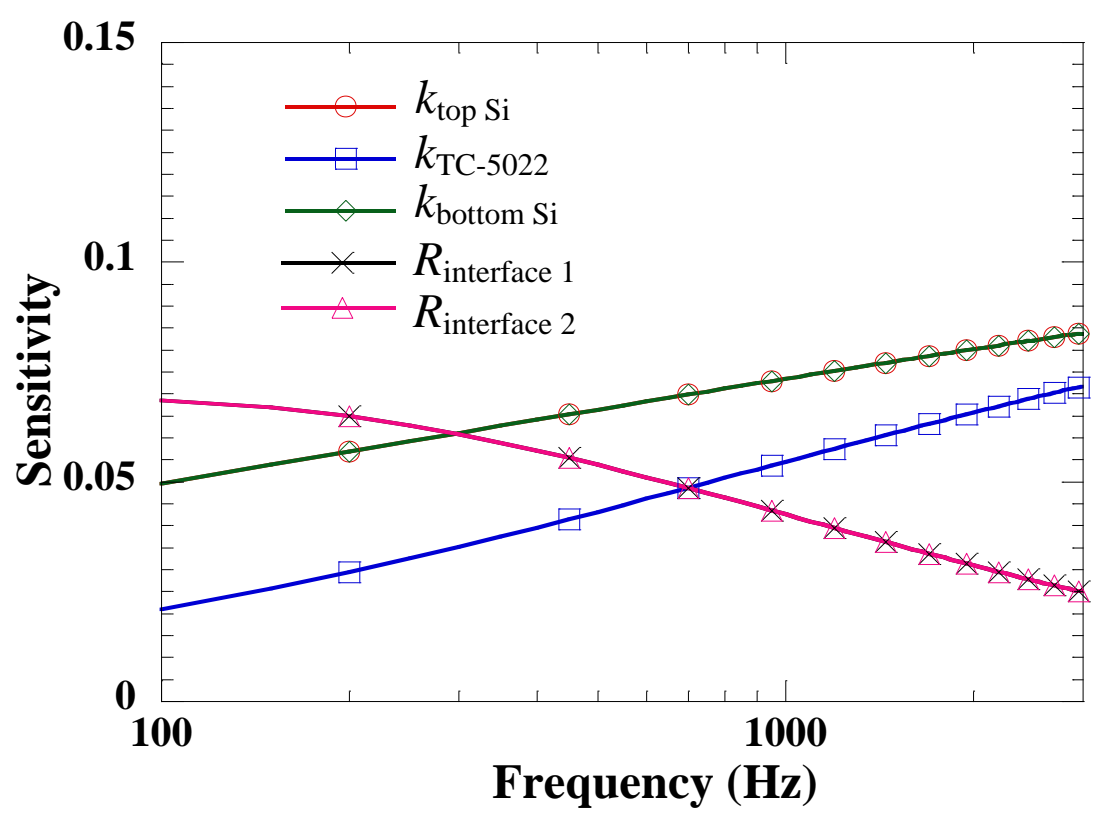

Fig. 7. Sensitivity of bulk thermal conductivity and contact resistance as a function of the frequency for the three-layer sample (TC-5022 grease sandwiched between silicon substrates).

\section{CONCLUSIONS}

In this work, we developed a general model to analyze the thermal energy transport in structures with $n$ number of layers. In contrast to the other general heat transfer models in the literature that require an assumption of a semi-infinite solid, this model can be used to estimate the heat transfer and consequent temperature gradient over a finite thickness. With proper assumptions of a planar heat source and 1-D heat transfer, we successfully derived general analytical solutions using a recursive matrix relationship to illustrate heat transfer through interfaces within the multilayer structure. This heat transfer model and the solutions provide insights into the experimental principles for the phase-sensitive thermoreflectance technique. The extracted phase information from the general solution involves a variety of thermal properties such as contact resistance and bulk thermal conductivity. With further analysis, a simplified 
general solution including only equivalent resistances from thermal paths that are equal to the thermal penetration depths in adjacent materials is finally achieved. Compared with solutions from other work in the literature, the solution proposed in the current work is simple and capable of delivering direct results of phase shift without complicated mathematical transforms and treatments. More importantly, the solution in this work provides clear and explicit insights into the physics and underlying mechanism that affect the heat transfer. It indicates that when a thermal wave passes a solid with interfaces or discontinuities, the thermal wave gets reflected at each interface. The multiple reflections accumulate and substantially complicate the heat transfer. Therefore, the analytical relation is highly coupled and nonlinear. Moreover, the contributions from reflections at interfaces and propagation through the bulk material can be drastically different depending on the applied frequency.

We validated this generic heat transfer model by applying the theoretical solutions to analyze the experimental data on typical samples using the PSTTR technique. We selected samples with different layers to comprehensively verify the capability and reliability of this model. These samples included single-layer plain silicon substrates, three-layer samples fabricated using commercial thermal grease, and a four-layer sample containing two aluminum-coated silicon substrates. We conducted PSTTR experiments on each of these samples with repetitions at different spots to minimize the random errors, along with calibration to reduce the system errors. While traditional techniques such as ASTM D5470 and laser flash are not capable of differentiating sources of thermal resistance within multilayer samples or measuring thermal resistances below $1 \mathrm{~mm}^{2} \cdot \mathrm{K} / \mathrm{W}$, PSTTR provides valuable information on the bulk thermal conductivity of the materials as well as the thermal contact resistance of the various interfaces. By applying the theoretical solutions to the PSTTR measurement data, the bulk thermal conductivity of the Dow Corning TC-5022 thermal grease (70 $\mu \mathrm{m}$ bond line thickness) was found to be $3.5 \mathrm{~W} /(\mathrm{m} \cdot \mathrm{K})$, the contact resistance was $8.7 \mathrm{~mm}^{2} \cdot \mathrm{K} / \mathrm{W}$, and the overall interfacial resistance $37.1 \mathrm{~mm}^{2} \cdot \mathrm{K} / \mathrm{W}$, showing good agreement with values from the literature and the measurements from the ASTM steady-state technique. The average overall thermal resistance of the direct-bonded sample was only $0.46 \mathrm{~mm}^{2} \cdot \mathrm{K} / \mathrm{W}$, lower than that of most known TIMs/BIMs. With the four-layer theoretical solution, we successfully determined the 
contact resistances of all the interfaces. The novel results showed that the contact resistance of the Al-Al interface is about one order of magnitude greater than that of the Al-Si interface. 


\section{ACKNOWLEDGMENTS}

The authors would like to acknowledge the support provided by Susan Rogers and Steven Boyd, Technology Development Managers for Electric Drive Technologies, Vehicle Technologies Office, U.S. Department of Energy Office of Energy Efficiency and Renewable Energy. The authors would like to acknowledge Ralph Taylor (Delphi) for providing the direct/diffusion-bonded sample. 


\section{REFERENCES}

[1] D.D.L. Chung, Thermal interface materials, Journal of Materials Engineering and Performance, 10(1) (2001) 56-59.

[2] R. Prasher, Thermal interface materials: Historical perspective, status, and future directions, Proceedings of the IEEE, 94(8) (2006) 1571-1586.

[3] F. Sarvar, D.C. Whalley, P.P. Conway, Thermal interface materials-A review of the state of the art, in: Electronics System Integration Technology Conference, Dresden, Germany, 2006, pp. 1292-1302.

[4] American Society for Testing and Materials, ASTM Standard D5470-01, 2005.

[5] W.J. Parker, R.J. Jenkins, C.P. Butler, G.L. Abbott, Flash method of determining thermal diffusivity, heat capacity, and thermal conductivity, Journal of Applied Physics, 32(9) (1961) 1679-1684.

[6] D.G. Cahill, Thermal-Conductivity Measurement from $30 \mathrm{~K}$ to $750 \mathrm{~K}$ : the 3-Omega Method, Review of Scientific Instruments, 61(2) (1990) 802-808.

[7] X.H. Feng, X.W. Wang, Thermophysical properties of free-standing micrometer-thick Poly (3hexylthiophene) films, Thin Solid Films, 519(16) (2011) 5700-5705.

[8] X.H. Feng, X.P. Huang, X.W. Wang, Thermal conductivity and secondary porosity of single anatase $\mathrm{TiO}_{2}$ nanowire, Nanotechnology, 23(18) (2012) 185701.

[9] X.H. Feng, X.P. Huang, X.W. Wang, Nonlinear effects in transient electrothermal characterization of anatase $\mathrm{TiO}_{2}$ nanowires, Review of Scientific Instruments, 83(4) (2012) 044901.

[10] C.A. Paddock, G.L. Eesley, Transient Thermoreflectance from Metal Films, Optics Letters, 11(5) (1986) 273-275.

[11] G.L. Doll, G.L. Eesley, M.S. Dresselhaus, G. Dresselhaus, A. Cassanho, H.P. Jenssen, D.R. Gabbe, Transient-thermoreflectance study of single-crystal lanthanum cuprate, Physical Review B, 40(13) (1989) 9354-9357.

[12] W.A. McGahan, K.D. Cole, Solutions of the heat conduction equation in multilayers for photothermal deflection experiments, Journal of Applied Physics, 72(4) (1992) 1362-1373. 
[13] Y. Fujii, A. Moritani, J. Nakai, Photoacoustic spectroscopy theory for multi-layered samples and interference effect, Japanese Journal of Applied Physics, 20(2) (1981) 361-367.

[14] H.P. Hu, X.W. Wang, X.F. Xu, Generalized theory of the photoacoustic effect in a multilayer material, Journal of Applied Physics, 86(7) (1999) 3953-3958.

[15] H.S. Carslaw, J.C. Jaeger, Conduction of Heat in Solids, second ed., Oxford University Press, Oxford, 1959, pp. 64-67.

[16] Y. Ohsone, G. Wu, J. Dryden, F. Zok, A. Majumdar, Optical measurement of thermal contact conductance between wafer-like thin solid samples, Journal of Heat Transfer, 121(4) (1999) 954-963.

[17] T. Tong, Y. Zhao, L. Delzeit, A. Kashani, M. Meyyappan, A. Majumdar, Dense vertically aligned multiwalled carbon nanotube arrays as thermal interface materials, IEEE Transactions on Components and Packaging Technologies, 30(1) (2007) 92-100.

[18] M.V. Iravani, H. Wickramasinghe, Scattering matrix approach to thermal wave propagation in layered structures, Journal of Applied Physics, 58(1) (1985) 122-131.

[19] R.J. Anderson, A method to calculate the laser heating of layered structures, Journal of Applied Physics, 64(12) (1988) 6639-6645.

[20] M.R. Madison, T.W. McDaniel, Temperature distributions produced in an N-layer film structure by static or scanning laser or electron beam with application to magnetooptical media, Journal of Applied Physics, 66(12) (1989) 5738-5748.

[21] A. Feldman, Algorithm for solutions of the thermal diffusion equation in a stratified medium with a modulated heating source, High Temperatures-High Pressures, 31(3) (1999) 293-298.

[22] D.G. Cahill, Analysis of heat flow in layered structures for time-domain thermoreflectance, Review of Scientific Instruments, 75(12) (2004) 5119-5122.

[23] Q. Hao, Analytical heat-transfer modeling of multilayered microdevices, Journal of Micromechanics and Microengineering, 14(7) (2004) 914-926.

[24] X. Feng, C. King, D. DeVoto, M. Mihalic, S. Narumanchi, Investigation of thermal interface materials using phase-sensitive transient thermoreflectance technique, in: Thermal and 
Thermomechanical Phenomena in Electronic Systems (ITherm), IEEE, Orlando, FL, 2014, pp. 1296-1307. [25] E. Marín, A. Lara-Bernal, A. Calderón, O. Delgado-Vasallo, On the heat transfer through a solid slab heated uniformly and continuously on one of its surfaces, European Journal of Physics, 32(3) (2011) 783791.

[26] J.A. Malen, K. Baheti, T. Tong, Y. Zhao, J.A. Hudgings, A. Majumdar, Optical measurement of thermal conductivity using fiber aligned frequency domain thermoreflectance, Journal of Heat Transfer, 133(8) (2011) 081601.

[27] N. Hmina, Y. Scudeller, Thermal interface resistance and subsurface effusivity of submicron metallic films on dielectric substrates: an experimental method for simultaneous determination, International Journal of Heat and Mass Transfer, 41(18) (1998) 2781-2798.

[28] F.P. Incropera, A.S. Lavine, D.P. DeWitt, Fundamentals of Heat and Mass Transfer, third ed., John Wiley \& Sons Incorporated, New York, 2011.

[29] A.S. Henry, G. Chen, Spectral phonon transport properties of silicon based on molecular dynamics simulations and lattice dynamics, Journal of Computational and Theoretical Nanoscience, 5(2) (2008) 141-152.

[30] http://www.dowcorning.com/content/publishedlit/11-1688a-01.pdf.

[31] S. Narumanchi, M. Mihalic, K. Kelly, G. Eesley, Thermal interface materials for power electronics applications, in: Thermal and Thermomechanical Phenomena in Electronic Systems (ITherm), IEEE, Orlando, FL, 2008, pp. 395-404.

[32] O.W. Kading, H. Skurk, K.E. Goodson, Thermal conduction in metallized silicon-dioxide layers on silicon, Applied Physics Letters, 65(13) (1994) 1629-1631.

[33] P.L. Komarov, M.G. Burzo, G. Kaytaz, P.E. Raad, Transient thermo-reflectance measurements of the thermal conductivity and interface resistance of metallized natural and isotopically-pure silicon, Microelectronics Journal, 34(12) (2003) 1115-1118. 
[34] P.E. Hopkins, J.R. Serrano, L.M. Phinney, Comparison of thermal conductivity and thermal boundary conductance sensitivities in continuous-wave and ultrashort-pulsed thermoreflectance analyses, International Journal of Thermophysics, 31(11-12) (2010) 2380-2393. 


\section{APPENDIX A}

In multilayer structure heat transfer model, a recursive coefficient matrix is established to connect the solution coefficient sets for different layers:

$$
\left(\begin{array}{l}
A_{i+1} \\
B_{i+1}
\end{array}\right)=D_{i} \cdot\left(\begin{array}{l}
A_{i} \\
B_{i}
\end{array}\right)
$$

in which $A_{i}, B_{i}, A_{i+1}$ and $B_{i+1}$ are coefficients in the solution for the adjacent layers $i$ and $i+1 . D_{i}$ is a $2 \times 2$ matrix and its elements are derived as follows:

$$
\begin{aligned}
& D_{11}=-S_{i i} \cdot S_{i+1, i}+C_{i i} \cdot C_{i+1, i} \frac{k_{i} \eta_{i}}{k_{i+1} \eta_{i+1}}-C_{i i} \cdot S_{i+1, i} \frac{k_{i} \eta_{i}}{h_{i, i+1}} \\
& D_{12}=-C_{i i} \cdot S_{i+1, i}+S_{i i} \cdot C_{i+1, i} \frac{k_{i} \eta_{i}}{k_{i+1} \eta_{i+1}}-S_{i i} \cdot S_{i+1, i} \frac{k_{i} \eta_{i}}{h_{i, i+1}} \\
& D_{21}=S_{i i} \cdot C_{i+1, i}-C_{i i} \cdot S_{i+1, i} \frac{k_{i} \eta_{i}}{k_{i+1} \eta_{i+1}}+C_{i i} \cdot C_{i+1, i} \frac{k_{i} \eta_{i}}{h_{i, i+1}} \\
& D_{22}=C_{i i} \cdot C_{i+1, i}-S_{i i} \cdot S_{i+1, i} \frac{k_{i} \eta_{i}}{k_{i+1} \eta_{i+1}}+S_{i i} \cdot C_{i+1, i} \frac{k_{i} \eta_{i}}{h_{i, i+1}}
\end{aligned}
$$

Here, we define $S_{i j}=\sinh \left(\eta_{i} \cdot b_{j}\right)$ and $C_{i j}=\cosh \left(\eta_{i} \cdot b_{j}\right)$ for simplification.

At $i=\mathrm{n}-1$, because of the geometric-relation constraints, we define $S_{n,(n-1), n}=\sinh \left[\eta_{n}\right.$. $\left.\left(b_{n-1}-b_{n}\right)\right]=-\sinh \left(\eta_{n} \cdot d_{n}\right)=-S_{n n}$, and $C_{n,(n-1), n}=\cosh \left[\eta_{n} \cdot\left(b_{n-1}-b_{n}\right)\right]=\cosh \left(\eta_{n} \cdot d_{n}\right)=$ $C_{n n}$. So, each element in the coefficient matrix of last interface, $D_{n-1}$, is slightly different from the one associated with other interfaces and all elements are also derived as:

$$
\begin{aligned}
& D_{11}=S_{n-1, n-1} \cdot S_{n, n}+C_{n-1, n-1} \cdot C_{n, n} \frac{k_{n-1} \eta_{n-1}}{k_{n} \eta_{n}}+C_{n-1, n-1} \cdot S_{n, n} \frac{k_{n-1} \eta_{n-1}}{h_{n-1, n}} \\
& D_{12}=C_{n-1, n-1} \cdot S_{n, n}+S_{n-1, n-1} \cdot C_{n, n} \frac{k_{n-1} \eta_{n-1}}{k_{n} \eta_{n}}+S_{n-1, n-1} \cdot S_{n, n} \frac{k_{n-1} \eta_{n-1}}{h_{n-1, n}} \\
& D_{21}=S_{n-1, n-1} \cdot C_{n, n}+C_{n-1, n-1} \cdot S_{n, n} \frac{k_{n-1} \eta_{n-1}}{k_{n} \eta_{n}}+C_{n-1, n-1} \cdot C_{n, n} \frac{k_{n-1} \eta_{n-1}}{h_{n-1, n}}
\end{aligned}
$$




$$
D_{22}=C_{n-1, n-1} \cdot C_{n, n}+S_{n-1, n-1} \cdot S_{n, n} \frac{k_{n-1} \eta_{n-1}}{k_{n} \eta_{n}}+S_{n-1, n-1} \cdot C_{n, n} \frac{k_{n-1} \eta_{n-1}}{h_{n-1, n}}
$$

With these two coefficient matrices, it is feasible to derive the coefficient set $A_{i}$ and $B_{i}$ for an arbitrary layer within the structure and consequently solve the temperature distribution.

\section{APPENDIX B}

To validate Eq. (10), we can assign specific values to the number of layers " $n$," for instance, 1, 2, and 3 , to derive the corresponding temperature distribution. When $n$ equals 2 , the two-layer case, the coefficients are

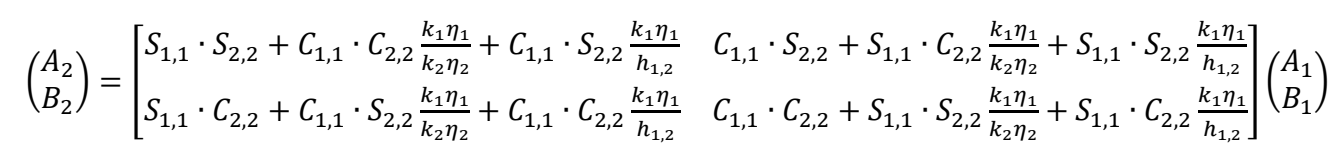

When $\mathrm{A}_{2}=0$, we then express the coefficient $B_{1}$ as

$$
B_{1}=\frac{S_{1,1} \cdot S_{2,2}+C_{1,1} \cdot C_{2,2} \frac{k_{1} \eta_{1}}{k_{2} \eta_{2}}+C_{1,1} \cdot S_{2,2} \frac{k_{1} \eta_{1}}{h_{1,2}}}{C_{1,1} \cdot S_{2,2}+S_{1,1} \cdot C_{2,2} \frac{k_{1} \eta_{1}}{k_{2} \eta_{2}}+S_{1,1} \cdot S_{2,2} \frac{k_{1} \eta_{1}}{h_{1,2}}} \cdot\left(-A_{1}\right)
$$

By substituting Eq. (B2) into the temperature distribution and conducting the inverse transforms under high-frequency assumption, it then becomes identical to the one in Ohsone et al.'s work [16, 24] after simplification.

If $n$ equals 3, we need to derive the matrices for two interfaces and they are,

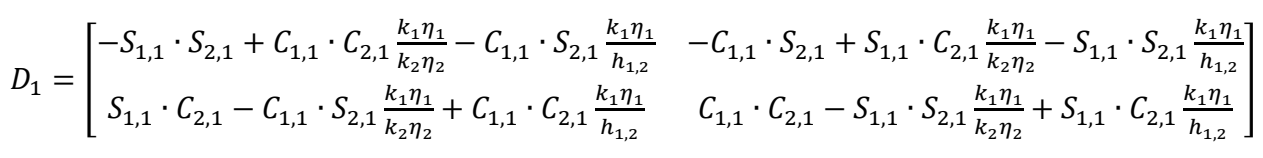

$$
\begin{aligned}
& D_{2}=\left[\begin{array}{ll}
S_{2,2} \cdot S_{3,3}+C_{2,2} \cdot C_{3,3} \frac{k_{2} \eta_{2}}{k_{3} \eta_{3}}+C_{2,2} \cdot S_{3,3} \frac{k_{2} \eta_{2}}{h_{2,3}} & C_{2,2} \cdot S_{3,3}+S_{2,2} \cdot C_{3,3} \frac{k_{2} \eta_{2}}{k_{3} \eta_{3}}+S_{2,2} \cdot S_{3,3} \frac{k_{1} \eta_{1}}{h_{1,2}} \\
S_{2,2} \cdot C_{3,3}+C_{2,2} \cdot S_{3,3} \frac{k_{2} \eta_{2}}{k_{3} \eta_{3}}+C_{2,2} \cdot C_{3,3} \frac{k_{1} \eta_{1}}{h_{1,2}} & C_{2,2} \cdot S_{3,3}+S_{2,2} \cdot S_{3,3} \frac{k_{2} \eta_{2}}{k_{3} \eta_{3}}+S_{2,2} \cdot C_{3,3} \frac{k_{1} \eta_{1}}{h_{1,2}}
\end{array}\right]
\end{aligned}
$$

and then the coefficient sets are: 


$$
\left(\begin{array}{l}
A_{3} \\
B_{3}
\end{array}\right)=D_{2}\left(\begin{array}{l}
A_{2} \\
B_{2}
\end{array}\right)=D_{2} \cdot D_{1}\left(\begin{array}{l}
A_{1} \\
B_{1}
\end{array}\right)=\left[\begin{array}{ll}
D_{11} & D_{12} \\
D_{21} & D_{22}
\end{array}\right]\left(\begin{array}{l}
A_{1} \\
B_{1}
\end{array}\right)
$$

As we have to multiply two matrices, we use MATLAB to calculate each element in the final matrix:

$$
\begin{aligned}
& D_{11}=S_{1,1} \cdot C_{2,2} \cdot S_{3,3}+C_{1,1} \cdot C_{2,2} \cdot S_{3,3} \frac{k_{1} \eta_{1}}{k_{2} \eta_{2}}+C_{1,1} \cdot C_{2,2} \cdot C_{3,3} \frac{k_{1} \eta_{1}}{k_{3} \eta_{3}}+C_{1,1} \cdot C_{2,2} \cdot S_{3,3} \frac{k_{1} \eta_{1}}{h_{1,2}}+C_{1,1} \cdot C_{2,2} \\
& S_{3,3} \frac{k_{1} \eta_{1}}{h_{2,3}}+S_{1,1} \cdot S_{2,2} \cdot C_{3,3} \frac{k_{2} \eta_{2}}{k_{3} \eta_{3}}+S_{1,1} \cdot S_{2,2} \cdot S_{3,3} \frac{k_{2} \eta_{2}}{h_{2,3}}+C_{1,1} \cdot S_{2,2} \cdot C_{3,3} \frac{k_{1} \eta_{1}}{h_{1,2}} \cdot \frac{k_{2} \eta_{2}}{k_{3} \eta_{3}}+C_{1,1} \cdot S_{2,2} \cdot S_{3,3} \frac{k_{1} \eta_{1}}{h_{1,2}} \\
& \frac{k_{2} \eta_{2}}{h_{2,3}} \\
& D_{12}=C_{1,1} \cdot C_{2,2} \cdot S_{3,3}+S_{1,1} \cdot S_{2,2} \cdot S_{3,3} \frac{k_{1} \eta_{1}}{k_{2} \eta_{2}}+S_{1,1} \cdot C_{2,2} \cdot C_{3,3} \frac{k_{1} \eta_{1}}{k_{3} \eta_{3}}+S_{1,1} \cdot C_{2,2} \cdot S_{3,3} \frac{k_{1} \eta_{1}}{h_{1,2}}+S_{1,1} \cdot C_{2,2} \cdot \\
& S_{3,3} \frac{k_{1} \eta_{1}}{h_{2,3}}+C_{1,1} \cdot S_{2,2} \cdot C_{3,3} \frac{k_{2} \eta_{2}}{k_{3} \eta_{3}}+C_{1,1} \cdot S_{2,2} \cdot S_{3,3} \frac{k_{2} \eta_{2}}{h_{2,3}}+S_{1,1} \cdot S_{2,2} \cdot C_{3,3} \frac{k_{1} \eta_{1}}{h_{1,2}} \cdot \frac{k_{2} \eta_{2}}{k_{3} \eta_{3}}+S_{1,1} \cdot S_{2,2} \cdot S_{3,3} \frac{k_{1} \eta_{1}}{h_{1,2}} \\
& \frac{k_{2} \eta_{2}}{h_{2,3}} \\
& D_{21}=S_{1,1} \cdot C_{2,2} \cdot C_{3,3}+C_{1,1} \cdot S_{2,2} \cdot C_{3,3} \frac{k_{1} \eta_{1}}{k_{2} \eta_{2}}+C_{1,1} \cdot C_{2,2} \cdot S_{3,3} \frac{k_{1} \eta_{1}}{k_{3} \eta_{3}}+C_{1,1} \cdot C_{2,2} \cdot C_{3,3} \frac{k_{1} \eta_{1}}{h_{1,2}}+C_{1,1} \cdot C_{2,2} \\
& C_{3,3} \frac{k_{1} \eta_{1}}{h_{2,3}}+S_{1,1} \cdot S_{2,2} \cdot C_{3,3} \frac{k_{2} \eta_{2}}{k_{3} \eta_{3}}+S_{1,1} \cdot S_{2,2} \cdot S_{3,3} \frac{k_{2} \eta_{2}}{h_{2,3}}+C_{1,1} \cdot S_{2,2} \cdot S_{3,3} \frac{k_{1} \eta_{1}}{h_{1,2}} \cdot \frac{k_{2} \eta_{2}}{k_{3} \eta_{3}}+C_{1,1} \cdot C_{2,2} \cdot C_{3,3} \frac{k_{1} \eta_{1}}{h_{1,2}} \\
& \frac{k_{2} \eta_{2}}{h_{2,3}}
\end{aligned}
$$

Because $A_{3}$ equals $0, A_{3}=D_{11} \cdot A_{1}+D_{12} \cdot B_{1}=0$. Therefore, $B_{1}=-\frac{D_{11}}{D_{12}} \cdot A_{1}$. In addition, $B_{3}$ is calculated as a function of coefficient $A_{1}$ :

$$
B_{3}=D_{21} \cdot A_{1}+D_{22} \cdot B_{1}=\frac{D_{21} \cdot D_{12}-D_{22} \cdot D_{11}}{D_{12}} \cdot A_{1}
$$


If the temperature variation at the bottom surface is of interest, solution for $w_{3}$ is:

$$
w_{3}=B_{3} \cdot \cosh \left[\eta_{3}\left(b_{3}-z\right)\right]
$$

Applying the inverse Hankel and Laplace transforms, we have:

$$
T_{3}(r, z, t)=\frac{q_{0} e^{-i \omega t}}{k_{1}} \int_{\lambda=0}^{\infty}\left[\frac{D_{21} \cdot D_{12}-D_{22} \cdot D_{11}}{D_{12}} \cdot \frac{\mathrm{H}(f(r))}{\eta_{1}} \cdot \cosh \left[\eta_{3}\left(b_{3}-z\right)\right]\right]_{s=-i \omega} \cdot \lambda J_{0}(\lambda r) d \lambda
$$

At the center of heat spot $(r=0)$ and bottom surface $\left(z=b_{n}\right)$, temperature distribution is further derived as:

$$
T_{3}\left(0, b_{3}, t\right)=\frac{q_{0} e^{-i \omega t}}{k_{1}} \int_{\lambda=0}^{\infty}\left[\frac{D_{21} \cdot D_{12}-D_{22} \cdot D_{11}}{D_{12}} \cdot \frac{\mathrm{H}(f(r))}{\eta_{1}}\right]_{s=-i \omega} \lambda d \lambda
$$

Therefore, by substituting Eq. (B5) into the integral in Eq. (B9) and further simplifying the expression, the temperature distribution is finally solved and its phase component is then extracted. 